\title{
BROADBAND PHYSICAL OPTICS SIMULATIONS OF LARGE MULTI-REFLECTOR ANTENNAS FOR RADIOASTRONOMY
}

\author{
Vladimir Yurchenko" ${ }^{1)}$ John Anthony Murphy ${ }^{1)}$, and Jean-Michel Lamarre ${ }^{2)}$ \\ "Department of Experimental Physics, National University of Ireland \\ Maynooth, Co. Kildare, Ireland \\ E-mail: v.yurchenko@may.ie, amurphy@may.ie \\ 2) Observatoire de Paris \\ 61 Av de l'Obsenvatoire, 75014, Paris, France \\ E-mail: jean-michel.lamarre@obspm.fr
}

\begin{abstract}
Challenges and solutions concerning the accurate electromagnetic simulations of large multi-reflector antennas for deep-space submillimeter-wave radioastronomy are considered. Current status of the ESA PLANCK HFI beam simulation is presented. Gaussian fitting of the computed beams is performed.
\end{abstract}

\section{Introduction}

With increasing the size and complexity of the spacebased antennas for radioastronomy, there is a demand for increasing the accuracy and efficiency of simulations of large multi-reflector telescope systems. A typical example is the ESA PLANCK Surveyor, the $3^{\text {rd }}$ generation deepspace dual-reflector submillimeter-wave telescope being designed for measuring the temperature anisotropies and polarization of the Cosmic Microwave Background (CMB).

Of two instruments on PLANCK, the High-Frequency Instrument (HFI) will cover the higher frequency channels centred at 100,143, 217, 353, 545 and $857 \mathrm{GHz}$ [1]. Four channels $(100-353 \mathrm{GHz})$ will use mono-mode quasiGaussian horns, half of them feeding polarization sensitive bolometers. The other two channels $(545$ and 857 $\mathrm{GHz}$ ) are feeding only non-polarized bolometers and use profiled multi-mode horns.

All the HFI horns are broadband, with the bandwidth of about $30 \%$ of central frequency. With the primary mirror diameter $D=1.5 \mathrm{~m}$, the electric size of the system $\mathrm{D} / \lambda$ extends up to 5000 . The system is designed to meet the extreme requirements on both the primary mirror edge taper $(-25-30 d B)$ and the angular resolution on the sky (about 5 arcminutes at the frequencies $217-857 \mathrm{GHz}$ ).

\section{Simulations of the Main Beams}

A challenge of electromagnetic simulations of large multi-reflector telescope systems is the need of a precise knowledge of main beams with extreme angular resolution (e.g., with the accuracy of power patterns better than $1 \%$ at the angular scales less than 1 arcminute) including also the knowledge of beam polarization in the form of the IQUV Stokes parameter patterns. Physical optics (PO) is the most adequate technique for this kind of simulations. Conventional software, however, cannot cope efficiently with the problems of this size.

As an alternative, we developed a unique ultra-fast $P O$ simulation method specifically designed for large asymmetric multi-reflector systems with broadband channels and complicated multi-mode structure of the source field.

The method allows us to perform fast and rigorous $\mathrm{PO}+\mathrm{PO} \mathrm{TE} / \mathrm{TM}$-mode simulations of the main beams of large telescopes such as PLANCK in a few minutes for mono-mode mono-frequency channels (e.g., on the sky mesh of $128 \times 128$ nodes with the convergence accuracy better than $0.1 \%$ ) and in about an hour for the broad-band polarization-averaged multi-mode channels (e.g., with nine frequencies and 16 spatial modes of the hom field in the highest PLANCK HFI frequency band $f=716-998 \mathrm{GHz}$ ).
We compute the beam patterns of the IQUV Stokes parameters on the sky by propagating the source field from the apertures of simulated CQM horns through the telescope mode-by-mode, with integration over the frequency band and with account of all polarizations of the field of non-polarized channels [2].

The latest broadband simulations of the PLANCK HFI beams provide the updates to the beam data published in [2]. There are five essential corrections to those data:

1) the horn design is slightly changed (mainly, due to the manufacturing procedure) so that the horns are slightly elongated compared to those used in [2],

2) the horn locations are now the final ones

3) both broadband and monochromatic beams are computed,

4) root-mean-square fitting is performed,

5) the gains of all mono-mode non-polarized beams in [2] should be increased by $3 \mathrm{~dB}$ (the gains of multi-mode horns in [2] are correct for those horns at those positions).

Typical results of simulations are shown in Figures 1, 2 .

\section{Gaussian Beam Fitting}

For the compact representation of the beam patterns, simulated broadband and mono-frequency beams are fitted by elliptical Gaussian functions similarly to Ref. [2]. The final results are also rather similar. Still, the difference of the respective patterns is, typically, about $1 \%$ due to the factors (1) - (5) mentioned above.

The fitting accuracy of mono-mode beams is found to be $1-2 \%$ for both the broadband and mono-frequency beams (the difference between the latter two patterns is also about $1 \%$ ). On the contrary, the multi-mode beams, being essentially flat-top, are much worse represented by simple Gaussian functions, with the error being up to $40 \%$ for both the broadband and mono-frequency beams.

As an example, Table 1 shows the updated Gaussian fitting parameters of the broadband beams HFI-143 (both polarized and non-polarized) defined similarly to Ref. [2] (broad frequency bands improve patterns).

Typical errors of beam fitting in all cases are rather symmetric that shows a possibility of a more advanced fitting providing a much higher accuracy while remaining sutficiently simple and compact.

Acknowledgement. This work was supported in part by the research grant from the Enterprise Ireland.

\section{References}

[1] Lamarre J.-M. et al. New Astronomy Reviews, 2003, v. 47, No 11-12, p. 1017-1024.

[2] Yurchenko V. B., Murphy J. A., Lamarre J.-M. and Brossard J. int. J. Intrared and Millimeter Waves, 2004, v. 25, No 4, p. $601-616$. 
Table 1. Gaussian fitting parameters of the broadband PLANCK HFI beams HFI-143 (cf. [2]; $f=121-165 G H z$, horns are placed at the final design positions, $W_{3 a b}$ is the full width of the original beam, $W_{F}=\left(W_{\max } W_{m i n}\right)^{1 / 2}$ and $G_{F}$ are the full width and the gain, respectively, of Gaussian fit of the same total power, $\varepsilon_{B}$ is the beam ellipticity, $\delta F$ is the fitting error and $\delta U, \delta V$ are the peak values of UV Stokes parameters)

\begin{tabular}{|c|c|c|c|c|c|c|c|c|c|c|c|c|}
\hline HFI beam & $\begin{array}{l}W_{\max } \\
\text { [arcmin] }\end{array}$ & $\begin{array}{c}W_{\min } \\
\text { [arcmin] }\end{array}$ & $\begin{array}{l}\varphi_{S C 0} \\
\text { [deg] }\end{array}$ & $\begin{array}{l}\eta_{s c o} \\
\text { [deg] }\end{array}$ & $\begin{array}{c}\tau \\
\text { [deg] }\end{array}$ & $\begin{array}{c}W_{3 d B} \\
{[\text { arcmin] }}\end{array}$ & $\begin{array}{c}W_{F} \\
{[a r c m i n]}\end{array}$ & $\begin{array}{c}G_{F} \\
{[d B i]}\end{array}$ & $\varepsilon_{B}$ & $\begin{array}{l}\delta F \\
\%\end{array}$ & $\begin{array}{l}\delta U \\
\%\end{array}$ & $\begin{array}{c}\delta V \\
\%\end{array}$ \\
\hline HFI-143-1a & 7.4323 & 6.8745 & 1.3718 & 6.1966 & 49.6927 & 7.30 & 7.15 & 63.51 & 1.081 & 2.1 & 0.9 & 4.0 \\
\hline HFI-143-1b & 7.4986 & 6.8574 & 1.3717 & 6.1966 & 49.0453 & 7.35 & 7.17 & 63.48 & 1.094 & 2.0 & 1.0 & 4.2 \\
\hline HFI-143-2a & 7.1939 & 6.9619 & 0.5637 & 6.2243 & 61.3503 & 7.29 & 7.08 & 63.62 & 1.033 & 2.2 & 0.6 & 3.7 \\
\hline $\mathrm{HFl}-143-2 b$ & 7.2439 & 6.9324 & 0.5637 & 6.2243 & 56.6716 & 7.32 & 7.09 & 63.60 & 1.045 & 2.2 & 0.5 & 3.8 \\
\hline HFI-143-3a & 7.2249 & 6.9377 & -0.5637 & 6.1992 & -61.7208 & 7.32 & 7.08 & 63.61 & 1.041 & 2.2 & 0.7 & 3.7 \\
\hline HFI-143-3b & 7.1967 & 6.9460 & -0.5637 & 6.1993 & -52.9134 & 7.29 & 7.07 & 63.62 & 1.036 & 2.2 & 0.5 & 3.8 \\
\hline HFI-143-4a & 7.5149 & 6.8715 & -1.4418 & 6.2210 & -51.4655 & 7.33 & 7.19 & 63.47 & 1.094 & 2.0 & 1.2 & 4.2 \\
\hline HFI-143-4b & 7.5083 & 6.8790 & -1.4419 & 6.2211 & -47.3673 & 7.33 & 7.19 & 63.47 & 1.091 & 2.0 & 1.0 & 4.3 \\
\hline HFI-143-5 & 7.5902 & 7.1702 & 1.1443 & 6.7334 & 62.8153 & 7.50 & 7.38 & 66.29 & 1.059 & 2.1 & 0.5 & 4.5 \\
\hline HFI-143-6 & 7.3981 & 7.1650 & 0.2997 & 6.7608 & 79.0498 & 7.41 & 7.28 & 66.41 & 1.033 & 2.0 & 0.5 & 4.2 \\
\hline HFI-143-7 & 7.3836 & 7.1576 & -0.2997 & 6.7358 & -78.5876 & 7.41 & 7.27 & 66.42 & 1.032 & 2.0 & 0.5 & 4.2 \\
\hline $\mathrm{HFI}-143-8$ & 7.6067 & 7.1833 & -1.1443 & 6.7585 & -63.2693 & 7.51 & 7.39 & 66.27 & 1.059 & 2.1 & 0.5 & 4.5 \\
\hline
\end{tabular}

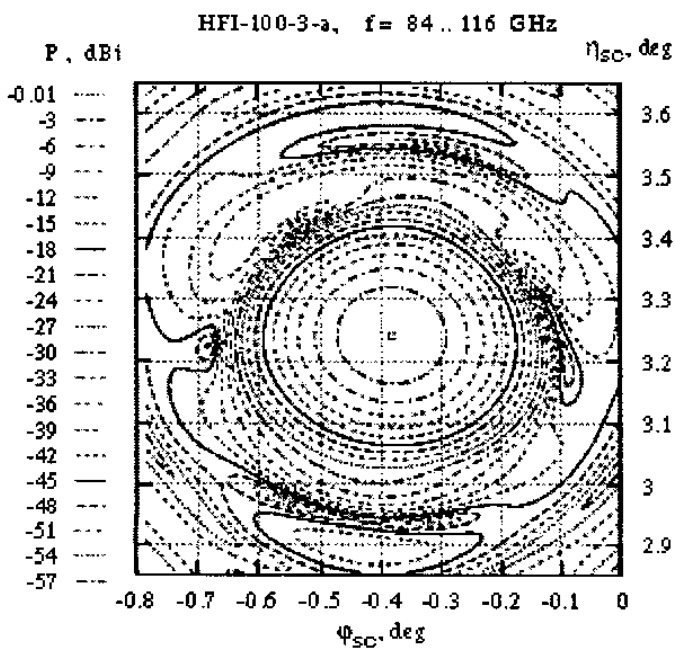

(a)

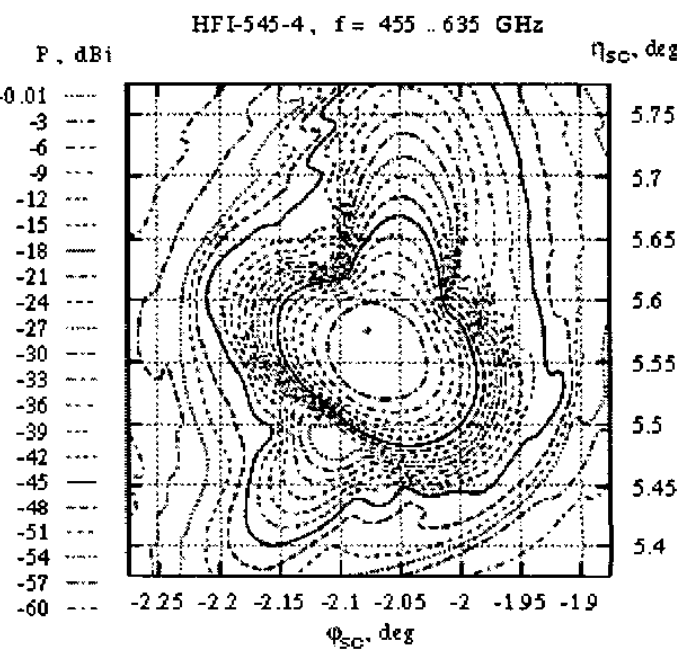

(b)

Figure 1. Power patterns of the broadband beams (a) HFI-100-3a (vertical polarization) and (b) HFI-545-4 (cf. [2])

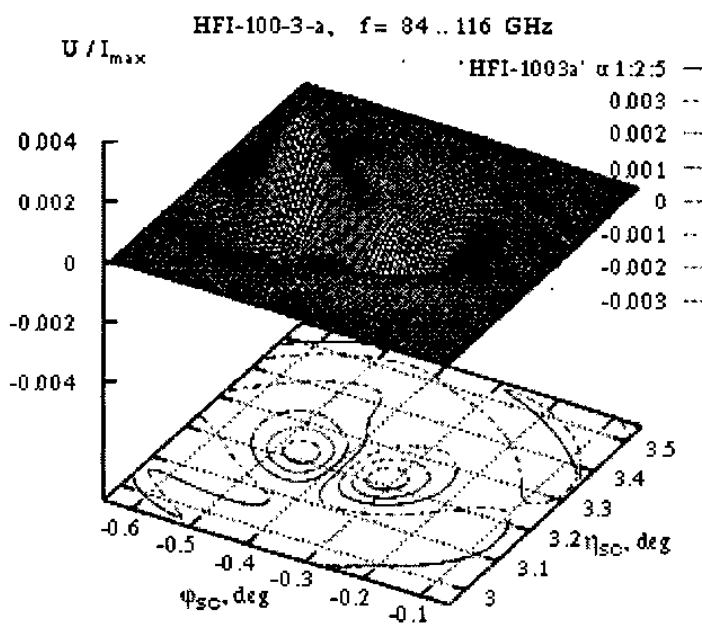

(a)

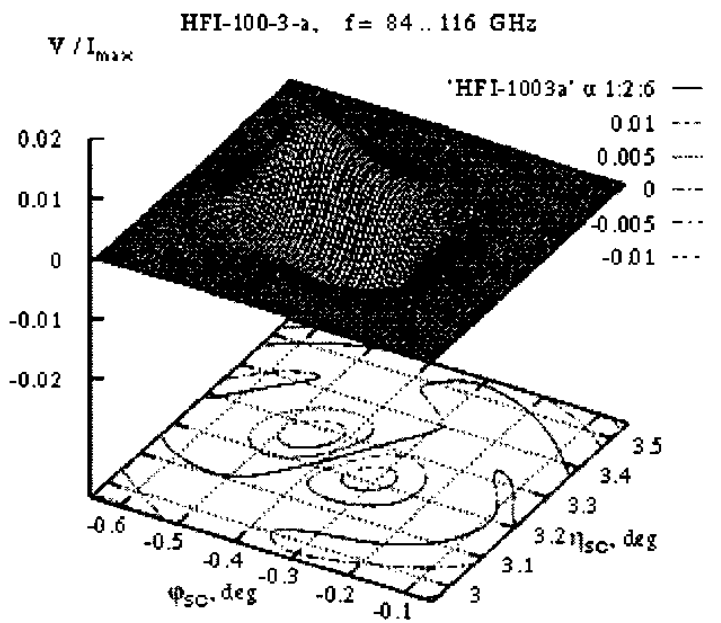

(b)

Figure 2. Broadband HFI-100-3a beam patterns of (a) $U$ and (b) $V$ Stokes parameters (vertical polarization, cf. [2]) 